\title{
Genetic and morphological diversity in sympatric kelps with contrasting reproductive strategies
}

\author{
M. A. Coleman ${ }^{1,2, *}$, T. Wernberg ${ }^{3}$ \\ ${ }^{1}$ Department of Primary Industries, NSW Fisheries, Coffs Harbour, NSW 2450, Australia \\ ${ }^{2}$ National Marine Science Centre, Southern Cross University, Coffs Harbour, NSW 2450, Australia \\ ${ }^{3}$ University of Western Australia Oceans Institute and School of Biological Sciences, University of Western Australia, \\ Crawley, WA 6009, Australia
}

\begin{abstract}
The evolution of asexual reproduction is considered a response to environmental conditions where it incurs less cost than sexual reproduction, maintains adapted genotypes and allows rapid proliferation into new areas. In rare circumstances, some species have evolved distinct asexual morphs or lineages in response to ubiquitous environmental conditions. Understanding the implications of, and the mechanisms underpinning, such reproductive strategies will be important for assessing the vulnerability of populations to environmental change. We examined morphological and genetic variation between 2 morphs of the kelp Ecklonia radiata, with sympatric haplodiplontic and vegetatively reproducing individuals growing side by side in Western Australia. Using 6 microsatellite markers, we show that vegetative morphs had a great propensity for asexual reproduction, with all attached haptera (8-20 per plant) being genetically identical to their parent plant. Moreover, for 8 multilocus genotypes (MLGs), Psex (probability that each MLG had clonal origins) scores were significant, suggesting clonal origins, and members of these MLGs were overwhelmingly dominated by individuals of the vegetative morph. Vegetative morphs were morphologically distinct, less morphologically variable, had lower genetic diversity and an excess of heterozygotes relative to haplodiplontic morphs. Nevertheless, vegetative morphs still produced and released zoospores at the same densities as haplodiplontic individuals, suggesting that they still complete an alternation of generations life history strategy. This likely accounted for weak genetic differentiation between morphs and suggests ongoing gene flow. Given that genetic diversity often confers adaptive capacity through change, low diversity may have implications for the vulnerability of this unique vegetative morph to local climatic and environmental stressors.
\end{abstract}

KEY WORDS: Kelp · Ecklonia radiata $\cdot$ E. brevipes $\cdot$ Dispersal $\cdot$ Gene flow $\cdot$ Genetic structure Subtidal reef $\cdot$ Connectivity $\cdot$ Asexual $\cdot$ Vegetative $\cdot$ Clonal

\section{INTRODUCTION}

In plants, the evolution of asexual reproduction is considered a response to environmental conditions where it incurs less cost than sexual reproduction, maintains adapted genotypes and allows rapid proliferation into new areas (see review by Holsinger 2000). Many species have haplodiplontic life cycles with alternate sexual and asexual stages (e.g. ferns, mosses and many algae), while others reproduce asexually via fragmentation, vegetative reproduction

${ }^{*}$ Corresponding author: melinda.coleman@gmail.com or stoloniferous growth, often under specific environmental conditions (e.g. seagrasses, some algae). In certain instances, however, species have evolved asexual reproductive strategies in response to different environmental conditions (asexual ecotypes) or in rare cases, under ubiquitous conditions, often with distinct morphologies (morphs). Understanding the implications of, and mechanisms underpinning, reproductive strategies will be important for assessing the vulnerability of species and populations to environmental change.

(C) The authors 2018. Open Access under Creative Commons by Attribution Licence. Use, distribution and reproduction are unrestricted. Authors and original publication must be credited. 
Asexual reproduction is common among marine algae and is part of a natural haplodiplontic alternation of generations life history strategy in many taxa. Similarly, many algal taxa can also reproduce asexually via clonal propagation of vegetative fragments, often allopatrically or under specific environmental conditions. The occurrence of unique asexual lineages in marine algae is often correlated with marginal habitats (Tatarenkov et al. 2005), limits of distribution (Oppliger et al. 2014), favourable conditions (Demes \& Graham 2011), range expansions (e.g. Kruger-Hadfield et al. 2016) or senescence (Westermeier et al. 2013, Murúa et al. 2017). Under such conditions, asexual reproduction is thought to be more advantageous, i.e. selected for (but see Oppliger et al. 2014) or is used as a mechanism to enhance survival (Pacheco-Ruíz et al. 2005) and promote rapid recovery (Murúa et al. 2017).

Kelps (Laminariales) have a haplodiplontic alternation of generations life cycle with sexual (gametophyte) and asexual (sporophyte) stages. Moreover, vegetative reproduction has also been documented in kelp sporophytes, but its prevalence, function and ecological significance are poorly understood. Vegetative reproduction in kelps can be stoloniferous or rhizoidal (e.g. from the holdfasts such as in Laminaria sinclairii, Demes \& Graham 2011, and in Ecklonia stolinifera, Bolton \& Anderson 1994) or from stipes (e.g. Macrocystis pyrifera integrifolia, Murúa et al. 2017) and occasionally from the thallus (E. maxima and E. radiata, Bolton \& Anderson 1994). Vegetative reproduction from the thallus of $E$. radiata occurs via the production of many small club-like haptera (rudimentary holdfasts) on the margins of secondary laminae of adult sporophytes (Fig. 1). What is particularly unusual about this mode of vegetative reproduction is the reversal in polarity of growth and meristematic tissue, with the apex of the thallus becoming the holdfasts of new individuals (Lindauer 1945). Although a similar mode of vegetative reproduction has also been observed occasionally in $E$. maxima from South Africa (Bolton \& Anderson 1994) and drift E. radiata from New Zealand (Lindauer 1945), what is unique about Australian E. radiata is that vegetatively produced haptera eventually make contact with the substratum, attach to rocks and are subsequently torn off the 'parent' plant, creating a new individual (Lindauer 1945, Lindauer et al. 1961; Fig. 1) and becoming a functional part of the local kelp forest community. Although this has been directly observed (authors' pers. obs.), factors such as the frequency of haptera attachment, fate and development of vegetatively produced plants and

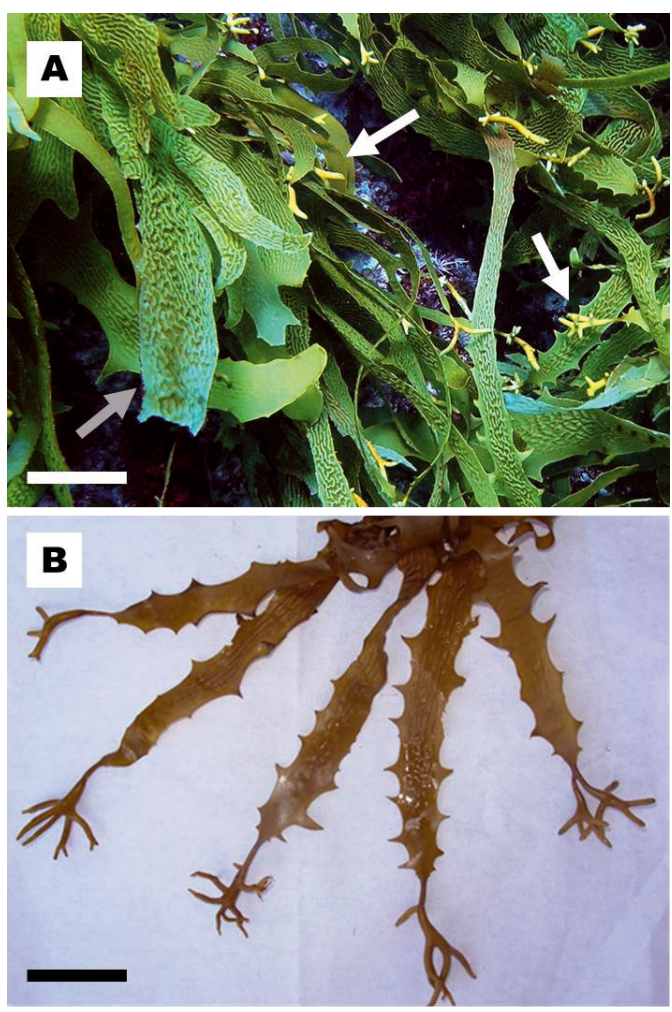

Fig. 1. Ecklonia radiata from Hamelin Bay, Western Australia. (A) Typical haplodiplontic morph (grey arrow) and vegetative morphs (formerly thought to be a separate species, E. brevipes; white arrows) grow side by side on the same reefs (scale bar $\sim 10 \mathrm{~cm}$ ). Haptera of the vegetative morph are unattached here. (B) Close-up of lateral ends of a vegetative morph showing well developed club-like haptera on the ends of secondary laminae (scale bar $\sim 5 \mathrm{~cm}$ )

their role in structuring local Ecklonia communities remain unknown.

Vegetative reproduction in Australian E. radiata appears to be largely restricted to the Cape Leeuwin area of Western Australia (authors' pers. obs.). Here, both haplodiplontic and vegetative morphs coexist in sympatry, interspersed side by side on the same limestone reefs (Fig. 1). However, the distribution of vegetative morphs is patchy among local reefs, with many reefs containing only the 'normal' haplodiplontic morph that is abundant throughout the entire temperate coastline of Australia (T. Wernberg pers. obs.). The mechanisms underpinning this sympatric distribution of morphs are intriguing and may provide insight into the evolution and functional significance of vegetative reproduction, as well as the vulnerability of this unique vegetative morph to environmental change. However, to date, there have been no genetic, demographic or ecological studies on this vegetative morph of E. radiata. 
Given the paucity of scientific information on this unique vegetative morph, here we characterise patterns of genetic, phenotypic and phenological variation of sympatric E. radiata morphs. Specifically, we (1) confirmed that reproduction is indeed vegetative (asexual) by comparing genotypes of adults with all attached haptera, (2) tested the hypothesis that genetic diversity of vegetatively reproducing morphs is low relative to reefs with only haplodiplontic morphs and (3) characterised phenotypic and phenological variation between sympatrically occurring morphs.

\section{MATERIALS AND METHODS}

Ecklonia radiata is the dominant kelp on subtidal reefs throughout Australasia (Bennett et al. 2016), where it plays a major role in structuring patterns of subtidal biodiversity (Coleman et al. 2007, Tuya et al. 2008). The usual mode of reproduction for E. radiata is a haplodiplontic alternation of generations life cycle whereby macroscopic sporophytes (spore-producing individuals) alternate with microscopic gametophytes (male and female gamete-producing individuals). Vegetatively reproducing sporophytes of E. radiata are only known from Hamelin Bay, Western Australia, and were formerly identified as a separate species, E. brevipes, also known from New Zealand (Huisman 2000). This classification was based exclusively on the presence of club-like haptera (small rudimentary holdfasts) that grow on the apex of the secondary laminae of adult plants, as well as clear visual differences in morphology (Fig. 1). Recent genetic studies, however, revealed that Australian E. radiata and $E$. brevipes are genetically similar and likely a single species (Rothman et al. 2015). Thus, here we recognise Australian E. brevipes as a vegetatively reproducing morph of $E$. radiata (Rothman et al. 2015) and have adopted the term 'vegetative morph' throughout. This is in contrast to the abundant and widely distributed $E$. radiata which we have termed here the 'haplodiplontic morph'.

\section{Genetic sampling}

To confirm asexual production of haptera and characterise genetic diversity and structure between morphs, E. radiata were collected from Hamelin Bay (34 13' 16.4" S, 11501' 42.4" E) in December 2006. Vegetative morphs were collected as fresh beach-cast wrack due to poor weather conditions at the time of sampling preventing diving on the offshore reefs where this vegetative morph occurs. Only intact plants with primary holdfasts and the presence of at least 1 asexual hapteron (as an identifier of the vegetative morph) were collected. Haplodiplontic E. radiata were sampled from 2 nearby accessible reefs (without the vegetative morph) that were $\sim 200 \mathrm{~m}$ apart in Hamelin Bay (Coleman et al. 2011b). For vegetatively reproducing individuals, all attached haptera developing on each adult individual, as well as a portion of the meristem of the 'parent' plant (identified by the presence of a large, fouled holdfast) were removed, rinsed in fresh water, blotted dry on paper towel and dried in silica gel for later genetic analyses. For haplodiplontic individuals, a clean, unfouled section of a secondary lamina was removed and treated as above.

DNA was extracted from a total of 66 vegetative morphs (identified via the presence of attached haptera). Of these, 12 were randomly chosen and DNA was extracted from all attached haptera (83 in total, 8 to 20 per parent plant) to confirm vegetative reproduction. DNA was also extracted from 67 haplodiplontic E. radiata as in Coleman et al. (2009). Microsatellite loci $(\mathrm{n}=6)$ developed for E. radiata (Dolman $\&$ Coleman 2009) were used to estimate genetic diversity and structure as in Coleman (2013). Prior to conducting statistical analyses, we checked data for typographical and genotyping errors, null alleles and stutter problems in the program MICROCHECKER (Van Oosterhout et al. 2004). Patterns of genetic diversity were characterised using a number of different descriptive measures. The total number of alleles and observed $\left(H_{\mathrm{o}}\right)$ and expected $\left(H_{\mathrm{e}}\right)$ heterozygosities were estimated using GENETIX ver. 4.04 (Belkhir et al. 2000). In addition, we tested for Hardy-Weinberg equilibrium at each locus and across all loci using FSTAT 1.2 (Goudet 1995). There was no evidence of linkage disequilibrium in any locus for E. radiata (Coleman et al. 2009, 2011a,b, Coleman 2013). Inbreeding and the extent of asexuality were estimated by testing $F_{\text {IS }}$ estimates using permutation tests (1000 permutations, FSTAT 1.2, Goudet 1995). Pairwise $F_{\mathrm{ST}}$ estimates were also estimated between the vegetative morph and 2 haplodiplontic reefs sampled. A sequential Bonferroni correction (Rice 1989) was used when examining significance levels for pairwise tests. We estimated the number of multilocus genotypes (MLGs) as well as Psex (the probability that each MLG had clonal origins) using GENECLONE (Arnaud-Haond \& Belkhir 2007) and calculated clonal richness as $R=(G-1) /(N-1)$ (Arnaud-Haond et al. 2007), where $\mathrm{N}$ is the number of sampling units and $\mathrm{G}$ is the number of genotypes. 


\section{Morphological and phenotypic sampling}

To characterise morphological and phenotypic differences within and between morphs of E. radiata, we sampled fully grown haplodiplontic and vegetatively reproducing E. radiata in April 2017, around the time of maximum thallus development (Wernberg \& Vanderklift 2010) and just prior to maximum fecundity (Mohring et al. 2013). Fifteen individuals of each morph were collected from 2 reefs $(\sim 100 \mathrm{~m}$ apart) within each of 2 sites (SH3 and $\mathrm{SH} 4 ; \sim 5 \mathrm{~km}$ apart) in Hamelin Bay. Collected individuals of each morph were growing interspersed (Fig. 1). While in the field, all individuals were kept moist and out of direct sunlight in large sacks. Once back on land, the sacks were stored overnight in a refrigerator $\left(5^{\circ} \mathrm{C}\right)$. Measurements of morphology and fecundity were made within $24 \mathrm{~h}$ of collection.

Fourteen morphological characters, described and defined by Wernberg et al. (2003), were measured on each individual: thallus length and weight; stipe length and diameter; central lamina twists, length, width and thickness; side lateral number, length, width, thickness and rugosity; and the number of laterals with vegetatively produced haptera. As the presence of lateral haptera was used as a defining character between the 2 morphs for sampling purposes, this character was not included in the analyses of morphological differences between the 2 morphs. Differences in morphology between morphs were tested by multivariate analyses based on normalised Euclidean distance dissimilarities calculated from untransformed data. Morphological differences were visualised through principal coordinates ordination (PCO), the contribution of different characteristics determined using SIMPER (Clarke 1993) and tested between species (fixed factor), sites (random factor) and areas nested within sites (random factor) by permutational multivariate analysis of variance (PERMANOVA), with 9999 permutations of the residuals under a reduced model (Anderson et al. 2008). Differences in morphological variability within morphs were tested by distance-based tests for homogeneity of multivariate dispersion (PERMDISP) with 9999 unrestricted permutations of residuals (Anderson et al. 2008) .

The possibility for both morphs to participate in haplodiplontic reproduction was tested by assessing the fecundity (production of spores) of vegetative morphs relative to haplodiplontic morphs. We did not assess the viability of spores by following the fate of gametophytes due to difficulties in culturing this species past the gametophyte stage. This was done by measuring zoospore release from sporophytes collected at the same 2 sites in February $2014(\mathrm{n}=10$ thalli morph ${ }^{-1}$ ) and April 2017 ( $\mathrm{n}=30$ thalli morph $^{-1}$ ) following the protocol of Mohring et al. (2013). Briefly, for each thallus, tissue discs were punched from the central lamina with a PVC corer, starting at the distal end of the plant. Following a $\sim 1 \mathrm{~h}$ desiccation period at ambient temperature, all discs from each thallus were placed in a single cup of seawater and gently stirred. After $20 \mathrm{~min}$, the zoospore solution was added to a vial with $0.1 \mathrm{ml}$ of $70 \%$ ethanol. Then, $1 \mathrm{ml}$ of the solution was placed onto a Neubauer counting chamber, and zoospores were counted in 6 grids sample ${ }^{-1}$. The results were averaged and converted to number of zoospores released per area of lamina sampled (total area of discs of tissue). Differences between morphs (fixed factor), dates (random factor) and sites (random factor) were tested by PERMANOVA on $\ln (x+1)$ transformed data, with 9999 permutations of the residuals under a reduced model (Anderson et al. 2008).

\section{RESULTS}

\section{Genetic patterns between morphs}

In plants collected for genetic analyses, there were between 2 and 20 adventitious haptera growing on the margins of each vegetative morph with (mean \pm SE) $8.59 \pm 0.54$ haptera thallus ${ }^{-1}$. All haptera on the 12 vegetative morphs that we examined had multilocus genotypes identical to those of the parent plant on which they were attached, confirming that haptera production was indeed asexual. Of the 66 vegetative-morph individuals sampled, we found 20 MLGs, with 9 shared between more than 1 individual ( 2 to 20 members per MLG). Psex for 5 of the shared MLGs was significant at $\mathrm{p}<0.01$, indicating clonal origins. For vegetative individuals, only locus K11 was in Hardy-Weinberg equilibrium.

We found genetic variability among vegetativemorph parent plants, and both homozygotes and heterozygotes were observed at each locus. However, there were fewer alleles over all loci among vegetative morphs (17 alleles, $\mathrm{n}=56$ individuals) relative to both nearby populations of haplodiplontic Ecklonia radiata (20 and 21 alleles at the 2 sites, respectively, $\mathrm{n}=35$ and 32 individuals; Table 1). Similarly, there were fewer MLGs (20) among the 66 vegetative parent plants sampled $(R=0.29)$ but 50 MLGs among the 67 haplodiplontic individuals sampled $(\mathrm{R}=0.74)$. For haplodiplontic morphs, only 8 MLGs were shared, 
Table 1. Total number alleles $\left(\mathrm{n}_{\mathrm{a}}\right)$, expected $\left(H_{\mathrm{e}}\right)$ and observed $\left(H_{\mathrm{o}}\right)$ heterozygosity and $F_{\text {IS }}$ for vegetative morphs $(\mathrm{n}=54)$ and 2 nearby haplodiplontic (HP) reproducing populations of Ecklonia radiata $(\mathrm{n}=35$ and 32) at each of 6 microsatellite loci. Significant $F_{\text {IS }}$ estimates after the Bonferroni correction are indicated in italics

\begin{tabular}{|c|c|c|c|c|c|c|c|c|c|c|c|c|}
\hline \multirow[t]{2}{*}{ Locus } & \multicolumn{4}{|c|}{ - Vegetative morph -} & \multicolumn{4}{|c|}{$\longrightarrow$ HP Site $1-$} & \multirow{2}{*}{$\mathrm{n}_{\mathrm{a}}$} & \multirow{2}{*}{$\bar{H}_{\mathrm{e}}$} & \multirow{2}{*}{$\begin{array}{c}\text { Site } 2 \\
H_{\mathrm{o}}\end{array}$} & \multirow[b]{2}{*}{$F_{\mathrm{IS}}$} \\
\hline & $\mathrm{n}_{\mathrm{a}}$ & $H_{\mathrm{e}}$ & $H_{\mathrm{o}}$ & $F_{\mathrm{IS}}$ & $\mathrm{n}_{\mathrm{a}}$ & $H_{\mathrm{e}}$ & $H_{\mathrm{o}}$ & $F_{\mathrm{IS}}$ & & & & \\
\hline K27 & 2 & 0.356 & 0.463 & -0.293 & 4 & 0.525 & 0.086 & 0.841 & 3 & 0.294 & 0.281 & 0.057 \\
\hline K30 & 2 & 0.036 & 0.000 & 1.000 & 3 & 0.182 & 0.029 & 0.848 & 3 & 0.200 & 0.031 & 0.848 \\
\hline K9 & 3 & 0.514 & 0.926 & -0.799 & 3 & 0.559 & 0.971 & -0.732 & 3 & 0.563 & 0.844 & -0.487 \\
\hline K10 & 5 & 0.561 & 0.574 & -0.015 & 5 & 0.620 & 0.429 & 0.322 & 5 & 0.655 & 0.406 & 0.393 \\
\hline K11 & 2 & 0.212 & 0.241 & -0.128 & 2 & 0.498 & 0.543 & -0.075 & 3 & 0.505 & 0.710 & -0.391 \\
\hline K32 & 3 & 0.526 & 1.000 & -0.899 & 4 & 0.540 & 1.000 & -0.848 & 3 & 0.603 & 1.000 & -0.649 \\
\hline Overall & 17 & 0.367 & 0.534 & -0.446 & 21 & 0.488 & 0.510 & -0.031 & 20 & 0.470 & 0.545 & -0.145 \\
\hline
\end{tabular}

with only 2 having significant $P$ sex values at $\mathrm{p}<0.01$. $F_{\text {IS }}$ was significantly negative in vegetative morphs, indicating an excess of heterozygotes among the parent plants (Table 1) and suggestive of asexual reproduction. This can be explained by the observed heterozygosity in vegetative morphs, which was significantly larger than expected over all loci (Table 1). These patterns appeared to be largely driven by 2 loci (K9 and K32) at which the vast majority of individuals exhibited the same heterozygous profile (129/131 at K9 and 192/194 at K32). Estimates of overall $F_{\text {IS }}$ were non-significant in both populations of haplodiplontic morphs, indicating random mating, but varied across loci (Table 1). However, $F_{\mathrm{IS}}$ estimates often deviated from random mating at individual loci, suggesting that there may be some amount of vegetative reproduction in the haplodiplontic populations sampled here (perhaps in the past), despite the visual absence of vegetative morphs at the time of sampling. Pairwise $F_{\mathrm{ST}}$ tests revealed weak but significant differentiation between the vegetative morph and both haplodiplontic sites sampled $\left(F_{\mathrm{ST}}=0.051\right.$ and 0.045 for Sites 1 and 2, respectively, $\mathrm{p}<0.05)$, but no differentiation between the 2 haplodiplontic sites $\left(F_{\mathrm{ST}}=0.0032\right)$.

\section{Morphological and phenological patterns}

Vegetative and haplodiplontic morphs had clearly distinct morphologies, and while there was random variation across sites and areas, by far the greatest proportion of variation was attributable to morphs (Table 2, Fig. 2). Of the 5 most important characters separating morphs, 4 were related to size and only 1 was related to shape (rugosity, Table 3). Sexual morphs were generally 2-4 times larger and had extensively rippled laterals compared to vegetative morphs, which were smaller and much smoother. In addition, the obvious distinction between morphs was that $49.8 \%$ of the laterals on vegetative morphs had haptera versus none of the haplodiplontic morphs. The level of morphological variation was $29.3 \%$ higher among haplodiplontic than vegetative morphs, as indicated by the dispersion around the centroid (PERMDISP, $F_{1,118}=40.7, \mathrm{p}($ perm $\left.)=0.0001\right)$.

Spores were successfully released from the primary laminae of the vegetative morph. Indeed, there were no differences in fecundity between morphs (pseudo- $F_{1,1.55}=9.93, \mathrm{p}=0.103 ;$ Fig. 3 ). There were also no differences in fecundity between sampling times or sites (PERMANOVA; $\mathrm{p}>0.05$ ). However,

Table 2. Results of PERMANOVA, testing differences in morphology of Ecklonia radiata morphs (fixed factor) from 2 reefs (random factor) nested in 2 sites (random factor). Bold indicates significance at $\mathrm{p}<0.05$. MC $=$ Monte Carlo $\mathrm{p}$-value

\begin{tabular}{|lccccccc|}
\hline Source & df & MS & Pseudo-F & p(perm) & perms & $\begin{array}{c}\text { p(MC) } \\
\text { \% components } \\
\text { of variation }\end{array}$ \\
\hline Morph & 1 & 747.26 & 39.215 & 0.2538 & 3 & $\mathbf{0 . 0 0 0 1}$ & 42.7 \\
Site & 1 & 33.774 & 2.5418 & 0.3263 & 3 & 0.0566 & 7.2 \\
Reef(Site) & 2 & 13.287 & 2.1405 & 0.005 & 9924 & $\mathbf{0 . 0 0 7 3}$ & 6.0 \\
Morph $\times$ Site & 1 & 19.056 & 1.5186 & 0.3015 & 800 & 0.2167 & 5.7 \\
Morph $\times$ Reef(Site) & 2 & 12.548 & 2.0214 & 0.0096 & 9906 & $\mathbf{0 . 0 1 1 2}$ & 8.0 \\
Residual & 112 & 6.2075 & & & & & 30.5 \\
\hline
\end{tabular}




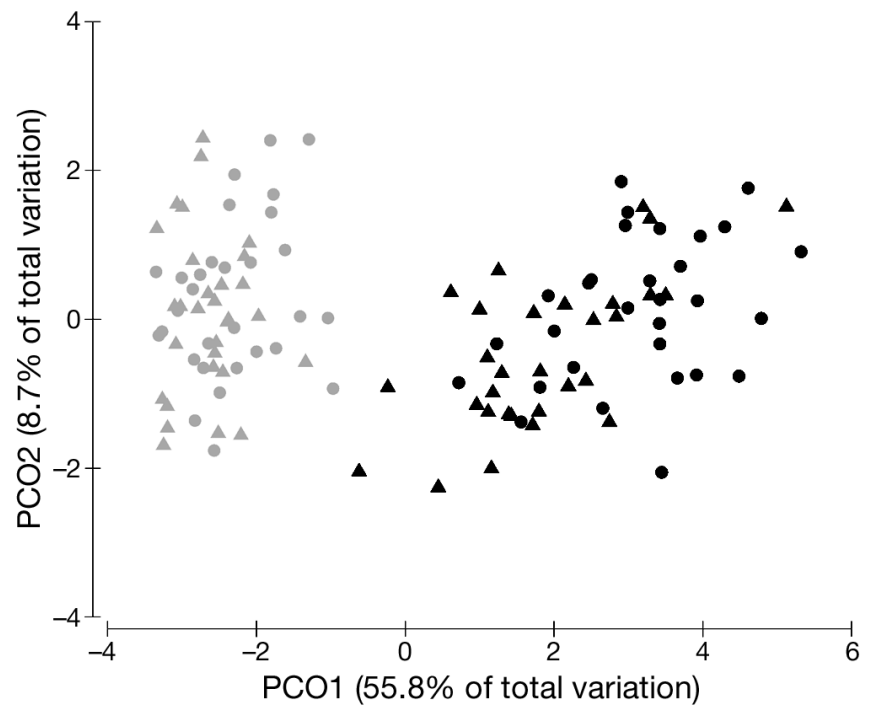

Fig. 2. Principal coordinates ordination (PCO) based on 13 morphological characters from vegetative (grey) and haplodiplontic (black) Ecklonia radiata morphs collected from 2 different sites ( $\boldsymbol{\Delta}$ vs. $\bullet$ ) in Hamelin Bay, Western Australia. The ordination is based on normalised Euclidian distances

Table 3. Results of SIMPER analysis showing the contribution of the 5 most important morphological characters to differences between morphs. The analysis was based on normalised morphological data and Euclidian distances

\begin{tabular}{|lccc|}
\hline $\begin{array}{l}\text { Morphological } \\
\text { character }\end{array}$ & $\begin{array}{c}\text { Haplo- } \\
\text { diplontic }\end{array}$ & $\begin{array}{l}\text { Vege- } \\
\text { tative }\end{array}$ & $\begin{array}{c}\text { \% Contr. } \\
\text { to diss. }\end{array}$ \\
\hline $\begin{array}{l}\text { Stipe diameter (mm) } \\
\text { Rugosity } \\
\text { (ripples cm }{ }^{-1} \text { ) }\end{array}$ & 12.1 & 6.7 & 9.37 \\
No. of laterals & 10.0 & 3.1 & 9.25 \\
Thallus fresh wt (g) & 88.2 & 22.9 & 9.12 \\
Lamina length (cm) & 88.3 & 41.3 & 8.92 \\
\hline
\end{tabular}

spore densities in both morphs $\left(<100 \mathrm{~mm}^{-2}\right)$ during this time (prior to the onset of the peak reproductive period) were on the low side of what can be seen during peak spore release (Mohring et al. 2013).

\section{DISCUSSION}

We investigated the genetic and demographic consequences of a unique vegetatively reproducing kelp morph that occurs in sympatry with haplodiplontic morphs. Production of haptera on vegetative morphs of Ecklonia radiata was confirmed to be asexual with between 8 and 20 genetically identical haptera produced on each parent plant. Given that these haptera

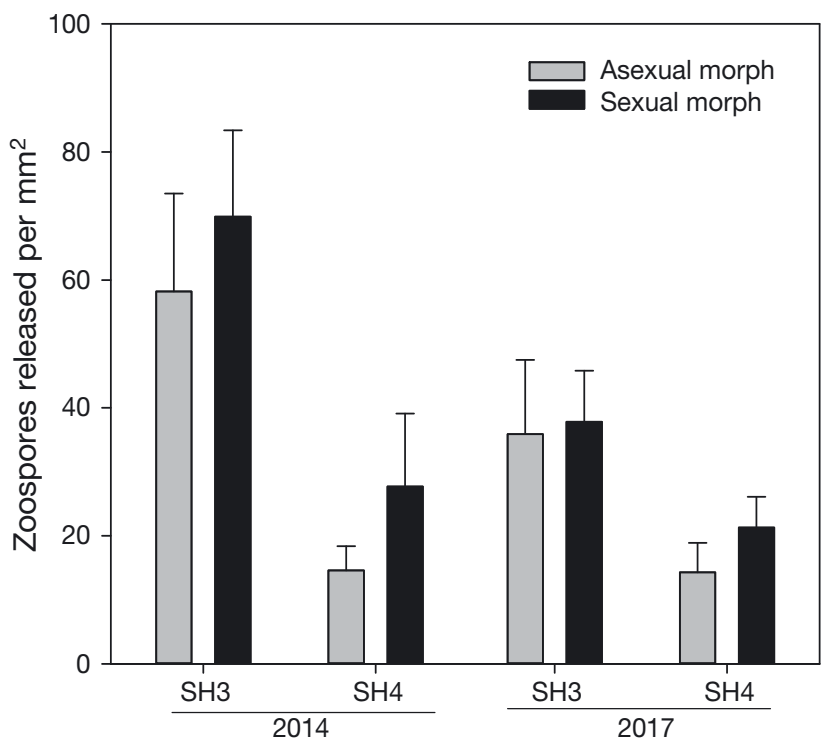

Fig. 3. Zoospore release density from haplodiplontic and vegetative sporophytes of Ecklonia radiata collected in February 2014 (n = 10 plants morph ${ }^{-1}$ ) and April 2017 (n = 30 plants $\mathrm{morph}^{-1}$ ) from each of 2 sites (SH3 and SH4). Data are mean $\pm \mathrm{SE}$

can attach to the substratum, detach from the parent plant and become a functional part of kelp communities (T. Wernberg pers. obs.), this represents a huge capacity for vegetative propagation, which was reflected in patterns of genetic diversity and structure as well as morphology of adult plants. Vegetative morphs were less morphologically variable, had lower genetic diversity (number of alleles and MLGs) and an excess of heterozygotes relative to nearby haplodiplontic morphs. Moreover, clonality was high $(\mathrm{R}=$ 0.29 ), and Psex estimates were significant for 5 MLGs (each with between 2 and 12 individual members), indicating clonal origins. This suggests that a founding subset of individuals may be largely responsible for continued vegetative reproduction resulting in increasing heterozygosity (Balloux et al. 2003), and that vegetative reproduction does not continually occur spontaneously among haplodiplontic morphs in nature. Given that genetic diversity often confers adaptive capacity through change (Reusch et al. 2005, Hughes et al. 2008, Wernberg et al. 2018), this lower diversity may have implications for the vulnerability of this unique morph to local climatic and environmental stressors, relative to co-occurring haplodiplontic morphs. Indeed, we recently documented the potential role of reduced genetic diversity in mediating vulnerability of $E$. radiata forests to climate stress at lower latitudes along this same coast (Wernberg et al. 2018). 
Despite genetic diversity (number of alleles) being lower in the vegetative morph than in nearby haplodiplontic morphs in Hamelin Bay, diversity estimates were similar to many other populations of haplodiplontic E. radiata throughout Australia (Coleman et al. 2011a,b, Coleman 2013). This raises the possibility that vegetative reproduction may be more prevalent than previously known, although we find this unlikely given the distinct morphology of vegetative morphs and their absence from extensive surveys elsewhere throughout Australia (Wernberg et al. 2003). Instead, the finding of only marginally lower diversity in vegetative morphs may be explained by the occurrence of sexual reproduction in vegetative morphs. Indeed, vegetative morphs still produced and released zoospores, and we found no differences in fecundity (spore production and release) between haplodiplontic and vegetative morphs. Although we did not assess the viability of subsequent gametophytes produced by vegetative morphs, nor their ability to successfully cross-fertilise with sexual gametes, there is likely some interbreeding which maintains gene flow between morphs. This is supported by the finding of only weak genetic differentiation between vegetative E. radiata and the 2 nearby sites in the same bay at which we sampled haplodiplontic morphs, indicating that there may be at least some ongoing gene flow between the 2 morphs.

Despite the huge propensity for vegetative propagation by E. radiata in Hamelin Bay (up to 20 genetically identical vegetative clones per adult plant), it is not known what proportion of these eventually attach to the substratum and become a functional part of local kelp communities. Certainly, it is common to see multiple haptera that are still attached to the parent plant wedged between rocks, as well as small putative vegetatively produced 'recruits' ( $\mathrm{T}$. Wernberg pers. obs.). Further, drift plants of the vegetative morph have been observed to lodge among rocks and can also putatively reattach at distant sites (M.A. Coleman pers. obs.), potentially creating 'founder effects' on small scales. Indeed, given the observation that haplodiplontic E. radiata morphs are continuously distributed on available rocky reef, whereas vegetative morphs are patchy $(\mathrm{T}$. Wernberg pers. obs), it is possible that founders locally propagate and spread within kelp forests. Our morphological data suggest that there are greater phenotypic differences in vegetative morphs between patches of reef ( $200 \mathrm{~m}$ apart) relative to co-occurring haplodiplontic morphs. Hierarchical sampling of genotypes within and among such patches would reveal whether there is small-scale spatial genetic structure and the realised propensity for vegetative morphs to locally proliferate, and this is currently being investigated.

Despite only weak genetic differentiation, haplodiplontic and vegetative morphs of $E$. radiata had vastly distinct morphologies. Apart from the defining characteristic of adventitious haptera, vegetative morphs were much smaller, smoother and had significantly lower biomass than haplodiplontic plants. It is possible that the size of vegetative individuals may be compromised by the cost of production of so many adventitious haptera. Regardless, the coexistence of these morphologically distinct morphs, both of which act as foundation species, has implications for local biodiversity and ecosystem functioning relative to more structurally homogeneous forests composed of only haplodiplontic morphs.

The mechanisms promoting this unique form of vegetative reproduction in sympatry with haplodiplontic E. radiata are intriguing. The species is not at the limits of distribution nor is it inhabiting what one might consider a marginal habitat (e.g. Tatarenkov et al. 2005, Oppliger et al. 2014). Similarly, the production of adventitious haptera on the apex of $\sim 50 \%$ of secondary laminae, along with vastly different morphologies and local occurrence of this morph, cannot be explained from a hypothesis of wounding or abrasion (McCook \& Chapman 1992) of haplodiplontic morphs. Asexual reproduction may be environmentally cued (e.g. Demes \& Graham 2011). For example, production of adventitious haptera may be induced in germlings or small sporophytes that are produced at a specific time of the year or under certain environmental conditions, but we find this unlikely given the localised extent of vegetative morphs in this kelp species. Auxin concentration often plays a role in algal development and polarity and may determine the production of vegetative haptera here (Ramakrishna \& De Smet 2014) as either a plastic response to environmental conditions (e.g. Kazan 2013) or as a heritable trait (e.g. mutation involving auxin genes; e.g. King et al. 1995). We contend that vegetative reproduction in $E$. radiata is most likely a heritable genetic characteristic (e.g. mutation) because of the sympatric distribution of morphs. Ecological studies that investigate the heritability of vegetative reproduction (e.g. reciprocal transplant experiments), as well as genetic studies that compare functional portions of the genome (e.g. RADSeq), will help answer these questions and provide important insight to understand and manage this unique morph into the future. 
Acknowledgements. This work was funded by an Australian Research Council Fellowship and Discovery grant to M.A.C. (DP0663550) and a Discovery grant (DP160100114) to T.W. and M.A.C. We thank B. P. Kelaher, C. Tuckett, Y. Mulders, S. Bell, M. Moustaka, N. Alsuwayan, M. Dahl and M. Mohring for assistance with field and laboratory work and G. Kendrick and 2 anonymous reviewers who greatly improved the manuscript.

\section{LITERATURE CITED}

Anderson M, Gorley R, Clarke K (2008) PERMANOVA+ for PRIMER: guide to software and statistical methods. PRIMER-E, Plymouth

Arnaud-Haond S, Belkhir K (2007) Geneclone: a computer program to analyse genotypic data, test for clonality and describe spatial clonal organization. Mol Ecol Notes 7: $15-17$

Arnaud-Haond S, Duarte CM, Alberto F, Serrão EA (2007) Standardizing methods to address clonality in population studies. Mol Ecol 16:5115-5139

Balloux F, Lehmann L, de Meeûs T (2003) The population genetics of clonal and partially clonal diploids. Genetics 164:1635-1644

Belkhir K, Borsa P, Chikhi L, Raufaste N, Bonhomme F (2000) GENETIX 402, logiciel sous Windows TM pour la génétique des populations. Université de Montpellier II, Montpellier

Bennett S, Wernberg T, Connell SD, Hobday AJ, Johnson CR, Poloczanska ES (2016) The 'Great Southern Reef': social, ecological and economic value of Australia's neglected kelp forests. Mar Freshw Res 67:47-56

Bolton J, Anderson R (1994) Ecklonia. In: Akatsuka I (ed) Biology of economic algae. SPB Academic Publishing, The Hague, p 385-406

Clarke KR (1993) Non-parametric multivariate analyses of changes in community structure. Austr J Ecol 18: $117-143$

Coleman MA (2013) Connectivity of the habitat-forming kelp, Ecklonia radiata within and among estuaries and open coast. PLOS ONE 8:e64667

Coleman MA, Vytopil E, Goodsell PJ, Gillanders BM, Connell SD (2007) Diversity and depth-related patterns of mobile invertebrates associated with kelp forests. Mar Freshw Res 58:589-595

Coleman MA, Gillanders BM, Connell SD (2009) Dispersal and gene flow in the habitat-forming kelp, Ecklonia radiata: relative degrees of isolation across an east-west coastline. Mar Freshw Res 60:802-809

* Coleman MA, Chambers J, Knott NA, Malcolm HA, Harasti D, Jordan A, Kelaher BP (2011a) Connectivity within and among a network of temperate marine reserves. PLOS ONE 6:e20168

* Coleman MA, Roughan M, Macdonald HS, Connell SD, Gillanders BM, Kelaher BP, Steinberg PD (2011b) Variation in the strength of continental boundary currents determines continent-wide connectivity in kelp. J Ecol 99:1026-1032

* Demes KW, Graham MH (2011) Abiotic regulation of investment in sexual versus vegetative reproduction in the clonal kelp Laminaria sinclairii (Laminariales, Phaeophyceae). J Phycol 47:463-470

Dolman G, Coleman MA (2009) Characterisation of microsatellite loci in the habitat-forming kelp, Ecklonia radi- ata (Phaeophyceae, Laminariales). Conserv Genet 10: $657-660$

Goudet J (1995) FSTAT (Version 1.2): a computer program to calculate $F$-statistics. J Hered 86:485-486

Holsinger KE (2000) Reproductive systems and evolution in vascular plants. Proc Natl Acad Sci USA 97:7037-7042

* Hughes AR, Inouye BD, Johnson MTJ, Underwood N, Vellend M (2008) Ecological consequences of genetic diversity. Ecol Lett 11:609-623

Huisman JM (2000) Marine plants of Australia. University of Western Australia Press, Nedlands

Krueger-Hadfield SA, Kollars NM, Byers JE, Greig TW and others (2016) Invasion of novel habitats uncouples haplodiplontic life cycles. Mol Ecol 25:3801-3816

Kazan K (2013) Auxin and the integration of environmental signals into plant root development. Ann Bot 112: 1655-1665

K King JJ, Stimart DP, Fisher RH, Bleecker AB (1995) A mutation altering auxin homeostasis and plant morphology in arabidopsis. Plant Cell 7:2023-2037

Lindauer VW (1945) Note on the brown alga Ecklonia brevipes J. Ag. Trans R Soc NZ 75:394-397

Lindauer VW, Chapman VJ, Aiken M (1961) The marine algae of New Zealand-II. Phaeophyceae. Nova Hedwigia 3:129-350

McCook LJ, Chapman ARO (1992) Vegetative regeneration of Fucus rockweed canopy as a mechanism of secondary succession on an exposed rocky shore. Bot Mar 35:35-46

* Mohring MB, Wernberg T, Kendrick GA, Rule MJ (2013) Reproductive synchrony in a habitat-forming kelp and its relationship with environmental conditions. Mar Biol 160:119-126

* Murúa P, Müller DG, Patiño DJ, Westermeier R (2017) Giant kelp vegetative propagation: adventitious holdfast elements rejuvenate senescent individuals of the Macrocystis pyrifera 'integrifolia' ecomorph. J Phycol 53:230-234

* Oppliger LV, von Dassow P, Bouchemousse S, Robuchon M and others (2014) Alteration of sexual reproduction and genetic diversity in the kelp species Laminaria digitata at the southern limit of its range. PLOS ONE 9:e102518

* Pacheco-Ruíz I, Zertuche-González JA, Espinoza-Ávalos J (2005) The role of the secondary attachment discs in the survival of Chondracanthus squarrulosus (Gigartinales, Rhodophyta). Phycologia 44:629-631

Ramakrishna P, De Smet I (2014) Evolutionary aspects of auxin signalling. In: Zažímalová E, Petrášek J, Benkova $\mathrm{E}$ (eds) Auxin and its role in plant development. Springer, Vienna, p 265-290

* Reusch TBH, Ehlers A, Hämmerli A, Worm B (2005) Ecosystem recovery after climatic extremes enhanced by genotypic diversity. Proc Natl Acad Sci USA 102:2826-2831

* Rice WR (1989) Analyzing tables of statistical tests. Evolution 43:223-225

Kothman MD, Mattio L, Wernberg T, Anderson RJ, Uwai S, Mohring MB, Bolton JJ (2015) A molecular investigation of the genus Ecklonia (Phaeophyceae, Laminariales) with special focus on the Southern Hemisphere. J Phycol 51:236-246

พ Tatarenkov A, Bergström L, Jönsson RB, Serrão EA, Kautsky L, Johannesson K (2005) Intriguing asexual life in marginal populations of the brown seaweed Fucus vesiculosus. Mol Ecol 14:647-651

* Tuya F, Wernberg T, Thomsen MS (2008) The spatial arrangement of reefs alters the ecological patterns of 
fauna between interspersed algal habitats. Estuar Coast Shelf Sci 78:774-782

Van Oosterhout C, Hutchinson WF, Wills DPM, Shipley P (2004) micro-checker: software for identifying and correcting genotyping errors in microsatellite data. Mol Ecol Notes 4:535-538

Wernberg T, Vanderklift MA (2010) Contribution of temporal and spatial components to morphological variation in the kelp Ecklonia (Laminariales). J Phycol 46:153-161

Wernberg T, Coleman M, Fairhead A, Miller S, Thomsen

Editorial responsibility: Nikolaos Schizas,

Mayagüez, Puerto Rico, USA
M (2003) Morphology of Ecklonia radiata (Phaeophyta: Laminarales [sic]) along its geographic distribution in southwestern Australia and Australasia. Mar Biol 143:47-55

*Wernberg T, Coleman MA, Bennett S, Thomsen MS, Tuya F, Kelaher BP (2018) Genetic diversity and kelp forest vulnerability to climatic stress. Sci Rep 8:1851

* Westermeier R, Murua P, Patino DJ, Munoz L, Ruiz A, Atero C, Mueller DG (2013) Utilization of holdfast fragments for vegetative propagation of Macrocystis integrifolia in Atacama, Northern Chile. J Appl Phycol 25:639-642

Submitted: November 30, 2017 ; Accepted: May 29, 2018

Proofs received from author(s): July 5, 2018 